\title{
Erratum to: Poor inhibitory control and neurochemical differences in high compulsive drinker rats selected by schedule-induced polydipsia
}

\author{
Margarita Moreno • Valeria Edith Gutiérrez-Ferre • \\ Luis Ruedas • Leticia Campa • Cristina Suñol • \\ Pilar Flores
}

Published online: 20 January 2012

(C) Springer-Verlag 2012

\section{Erratum to: Psychopharmacology}

$$
\text { DOI 10.1007/s00213-011-2575-y }
$$

There is an error in Table 2. The data for Striatum Control group: Ratio (DOPAC + HVA)/DA is 0.18 , and not 0.02 . Below is the corrected Table 2.

Table 2 Moanoamine concentration levels (picomole/milligramme of tissue) in the prefrontal cortex, striatum, nucleus accumbens and amygdala in unexposed and exposed SIP rats

\begin{tabular}{|c|c|c|c|c|c|c|c|c|}
\hline & \multicolumn{2}{|l|}{$\mathrm{PFC}$} & \multicolumn{2}{|l|}{ Striatum } & \multicolumn{2}{|l|}{ NAc } & \multicolumn{2}{|l|}{ Amyg } \\
\hline & Control & SIP & Control & SIP & Control & SIP & Control & SIP \\
\hline $\mathrm{NE}$ & $1.32 \pm 0.06$ & $1.26 \pm 0.05$ & $1.08 \pm 0.08$ & $1.07 \pm 0.04$ & $8.27 \pm 0.52$ & $5.44 \pm 0.30^{\mathrm{a}}$ & $2.15 \pm 0.15$ & $1.71 \pm 0.13^{\mathrm{a}}$ \\
\hline \multicolumn{9}{|c|}{ 5-HIAA/5HT } \\
\hline \multicolumn{8}{|c|}{ (DOPAC+HVA)/DA } & $0.76 \pm 0.02$ \\
\hline Ratio & $5.11 \pm 1.74$ & $1.54 \pm 0.16^{\mathrm{a}}$ & $0.18 \pm 0.01$ & $0.20 \pm 0.01$ & $0.24 \pm 0.02$ & $0.29 \pm 0.01^{\mathrm{a}}$ & $0.20 \pm 0.03$ & $0.18 \pm 0.01$ \\
\hline
\end{tabular}

Data are mean \pm SEM

$P F C$ prefrontal cortex, NAc nucleus accumbens, Amyg amygdala

${ }^{a}$ Statistical analyses indicate significant differences between control and SIP groups

The online version of the original article can be found at http://dx.doi. org/10.1007/s00213-011-2575-y.

M. Moreno · V. E. Gutiérrez-Ferre $\cdot$ L. Ruedas $\cdot$ P. Flores $(\bowtie)$

Departamento de Neurociencia y Ciencias de la Salud,

Universidad de Almería,

Carretera de Sacramento s/n, La Cañada de San Urbano,

04120, Almería, Spain

e-mail: pflores@ual.es

L. Campa $\cdot$ C. Suñol

Instituto de Investigaciones Biomédicas de Barcelona,

IIBB-CSIC-IDIBAPS, CIBERESP (CS), CIBERSAM (LC),

Barcelona, Spain 